\title{
Sparse signal reconstruction based on experimental chaos generated by a laser diode
}

\author{
D. Rontani, ${ }^{\mathrm{a}, \mathrm{b}, *}$ D. Choi,,${ }^{\mathrm{c}, \mathrm{d}}$ C.-Y. Chang, ${ }^{\mathrm{c}, \mathrm{e}}$ A. Locquet, ${ }^{\mathrm{c}, \mathrm{d}, \dagger}$ and D.S. Citrin ${ }^{\mathrm{c}, \mathrm{d}}$ \\ aOPTEL Research Group, CentraleSupélec, Université Paris-Saclay, F-91192 Gif-sur-Yvette, France \\ iL \\ ${ }^{c}$ UMI 2958 Georgia Tech - CNRS, Georgia Tech Lorraine, 2-3 Rue Marconi 57070 Metz, France \\ ${ }^{\mathrm{d}}$ Georgia Institute of Technology, School of Electrical and Computer Engineering, Atlanta, GA USA \\ ${ }^{\mathrm{e}}$ Georgia Institute of Technology, School of Physics, Atlanta, Georgia, USA
}

\begin{abstract}
We demonstrate experimentally that optical chaos generated by laser diode with optical feedback is suitable for compressive sensing of sparse signals. Specifically, we find that a wide range of the laser operational parameters and dynamical complexity guarantees that the generation of a sensing matrix, necessary for sparse reconstruction, has a comparable level of performance to those constructed with Gaussian random sequences. Our result opens new avenues for the use of optical chaotic devices for signal processing applications at ultra-high speed.
\end{abstract}

Keywords: Information Processing, Optical chaos, time-delay systems, semiconductor lasers, nonlinear dynamics

\section{INTRODUCTION}

Reconstructing quasi-perfectly sparse signals (i.e. equal to zeros most of the time) sampled below the ShannonNyquist limit is possible. Typically, for a baseband signal with total bandwidth $2 \mathrm{~B}$ it is necessary to sample the signal with a frequency $F_{S} \gg 2 B$ to reconstruct perfectly the signal. This is known as the Shannon-Nyquist limit. However, in the case of sparse signal, the informative content of the signal can be very limited with respect to the occupied bandwidth; hence, it is possible to exploit this property to design advanced reconstruction algorithm. This is known as compressive sensing (CS) [1-3].

The CS technique has found many applications in various fields of image processing and in applied physics. For example, this has been used in medical imaging to improve acquisition of MRI data [4], in quantum mechanics to reconstruct the density operator matrix [5], and in optics for holographic reconstruction [6].

To achieve CS, the sparse signal of interest is projected on a basis of vectors with component constructed with random variables. This is called sensing. Based on theory developed by E. Candès and T. Tao [1,2]. To achieve the best level of performance for sensing, it is known that independent identically distributed (iid) Gaussian random variables are a suitable choice.

The apparent randomness of optical chaos, although it does not have Gaussian statistics, has been harnessed in information processing such as secure communications [7] and the generation of true random numbers [8]. Here, we aim at realizing a sensing mechanism using optical chaos rather than numerically generated Gaussian random numbers.

In this study, we propose to use experimental measurements of the optical intensity generated by a laser diode subjected to optical feedback in its coherence collapse regime for the sensing mechanism. We show on a typical benchmarking problem from CS that it is possible to reconstruct sparse signal using chaotic samples generated by our chaotic laser with reconstruction performance close to identical to those of using Gaussian random variables, although

\footnotetext{
*damien.rontani@centralesupelec.fr

† alocquet@georgiatech-metz.fr
} 
our chaos-based sensing mechanism has statistical and spectral properties that are very different to those considered in the optimal case.

\section{EXPERIMENT AND CHAOS-BASED SENSING}

In our experimental setup, which is illustrated in Fig.1, we use a single-longitudinal-mode DFB laser diode with an emission centered at $1550 \mathrm{~nm}$. The laser is subjected to optical feedback, which introduces a delay in the dynamics of approximately $\tau=4.3 \mathrm{~ns}$. In the external cavity, we have introduced a polarizer and a quarter-wave plate to control the amount of light fed back in the laser cavity. The maximum feedback strength accounts for approximately $20 \%$ of the total optical power of the laser diode (measured to be around $15 \mathrm{~mW}$ ). Finally, to sample and acquire the optical chaotic time series, we use an amplified photodiode NewFocus $1544-\mathrm{B}$ with a $12 \mathrm{GHz}$ bandwidth, and a fast oscilloscope Agilent DSO 80804B with $12 \mathrm{GHz}$ bandwidth. This setup is identical to the one used in Ref. [9].

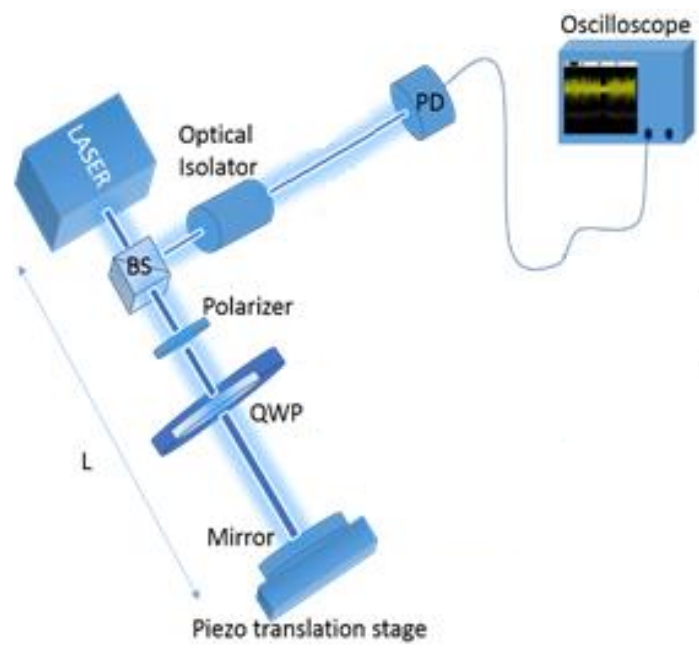

Figure 1 - Experimental setup of an external-cavity semiconductor laser (ECSL) for the generation of chaotic time-series. The optical feedback is composed a polarizer, a quarter wave-plate (QWP), which both allow to control the feedback strength and of a mirror mounted on a translation stage to adjust the feedback phase. The ECSL intensity is detected by a NewFocus photodiode (PD) and digitized by a fast oscilloscope.

In our experiment, the chaotic intensity time-series is sampled with period $T_{\mathrm{s}}=25 \mathrm{ps}$ and is converted to a digital representation with an 8-bits precision (see Fig. 2(a) for an example of intensity time series). We first analyze the spectral properties of our digitized time series, and evaluate the necessary decorrelation time that guarantees consecutive samples are statistically uncorrelated. In this study, we consider the following experimental parameters: $J=1.2 J_{\text {th }}$, where $J_{\text {th }}$ is the threshold of the free-running laser and feedback strength $\eta=12 \%$ of the total optical power. For this particular set of parameters, we compute the autocorrelation function (ACF) defined by

$$
A C F(\theta)=\frac{1}{\sigma_{I}}\left\langle I(t+\theta)-\mu_{I}\right\rangle\left\langle I(t)-\mu_{I}\right\rangle
$$

where $\mu_{I}=\langle I(t)\rangle$ is the time average of the intensity time series and $\sigma_{I}=\left\langle\left(I(t)-\mu_{I}\right)^{2}\right\rangle^{1 / 2}$ is the standard deviation of the time series. The evolution of the autocorrelation function is shown in Fig. 2. The time-lag values for which the correlation between samples is approximately zero corresponds to $\theta=T_{\text {sub-s }}=50 T_{\mathrm{s}}=1.25 \mathrm{~ns}$. With such decorrelation properties, the chaotic samples are iid, which is one of the requirements to generate suitable sensing matrices according to CS theory. Interestingly, time-delay systems such as ECSLs can display, under certain experimental conditions, relatively strong revival of their correlation for time lags which are integer multiple of the time delay $\tau$ [10,11]. Over the past decade, this phenomenon has been exhaustively documented in various photonic devices [12-13]. However, because of the particular choice of construction of the sensing matrix explained below this does not constitute a practical problem 
and only the decorrelation at time lag close to zero is important. Another way to circumvent the correlation signatures of the time delay is to resample the time trace with a sub-sampling factor so that the ratio $\tau / T_{\text {sub-s }}$ is not an integer. For example, with our current sampling precision, we have $\tau=182 T_{\mathrm{s}}$ and $T_{\text {sub-s }}=50 T_{\mathrm{s}}$.

The samples are used to form the sensing matrix later used in CS. We arrange the consecutive sampled by column of size $M$. We stop the construction of the matrix when we reach $N>>M$ columns, where $N$ is the size of the sparse signal to be reconstructed and $M$ corresponds to the number of measurements. Such a construction for the chaosbased sensing matrix is reminiscent of the method used in Ref. [14], where a logistic map was numerically implemented for the generation of chaotic samples.
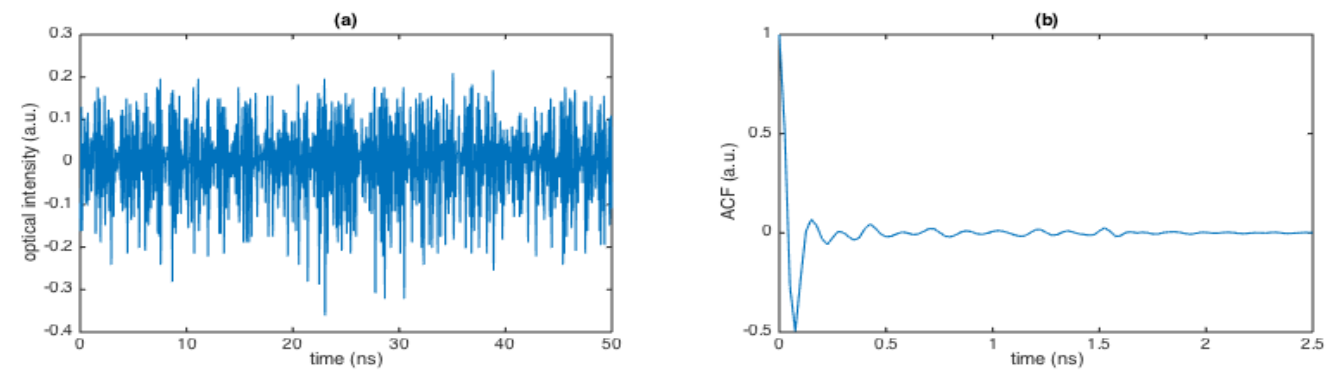

Figure 2 - (a) Experimental time series generated by the semiconductor laser with optical feedback with $J=1.2 J_{\text {th }}$ and $\eta=$ $12 \%$. (b) Autocorrelation function of the experimental intensity time series. The autocorrelation shows oscillations associated with the undamped relaxation oscillations and is identically equal to zero for time lags

In the CS theory, we generate $M$ measurements by realizing the scalar product between the unknown sparse signal $x$ to be reconstructed and the $M$ rows of the sensing matrix $\Phi$. This can be mathematically described by the following relationship $y=\Phi x$. This mathematical operation realizes a projection on a lower-dimensional subspace such that the amount of information about $x$ is significantly reduced. However, if the sensing matrix has the so-called restrictive isometry property (which is satisfied by Gaussian random matrices), it is possible to reconstruct quasiperfectly the original sparse signal with a high probability.

The reconstruction in the framework of CS is achieved with the resolution of a linear optimization problem with linear constraints. Mathematically, this reads

$$
\hat{x}=\min _{x}\|x\|_{L_{1}} \text { with } \quad y=\Phi x,
$$

where $\|\cdot\|_{L_{1}}$ denoting the measurement with the chaotic sensing matrix is the linear constraint in the linear program. We solve this linear program using the L1-Magic Toolbox developed by Candès and Romberg [16].

\section{RESULTS}

To demonstrate the performance of CS using chaotic sequence generated by an external cavity semiconductor laser, we consider a standard benchmark known as the basis pursuit problem. This consists of generating a sparse signal of size $N$ comprising $K<N$ non-zeros values distributed randomly and with values also taken randomly in the set $\{-1,1\}$ with equal probability $1 / 2$. In this benchmark, we apply the compressive sensing reconstruction algorithm to sparse signal with decreasing sparsity (increasing values of $\mathrm{K}$ ) and evaluate the probability of successful reconstruction. The threshold of accurate reconstruction is determined by having a relative error between the original signal and reconstructed signal $\epsilon=$ $\frac{\|\hat{x}-x\|}{\|x\|}$ less than 0.01 . At a given value of $M$, there exists a maximum level of sparsity so that the probability of accurate reconstruction drops rapidly. 
We realize a first experiment at high and low sparsity levels $K=5$ and $K=40$, respectively, for a signal of size $N$ $=100$ and $M=50$ measurements realized with our chaos-based sensing matrix. The results are shown in Fig. 3. In Fig. 3(a1)-(a2), we have represented the original sparse signal used in the basis pursuit problem, in Fig. 3(b1)-(b2), the reconstructed signal, and in Fig. 3(c1)-(c2), the reconstruction error between the two signals. In the case of accurate reconstruction, the relative error is far below the upper bound for success and the spiky structure of the original signal is well preserved. On the other hand, when the sparsity is low, we observe that the reconstruction did not preserve the spiky structure at all leading to an important relative error. The results would have been similar with a sensing matrix based on Gaussian random variables.
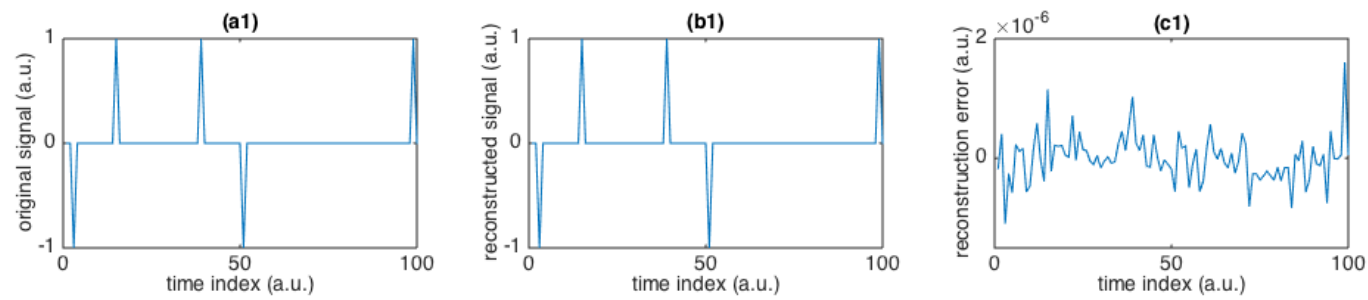

(a2)
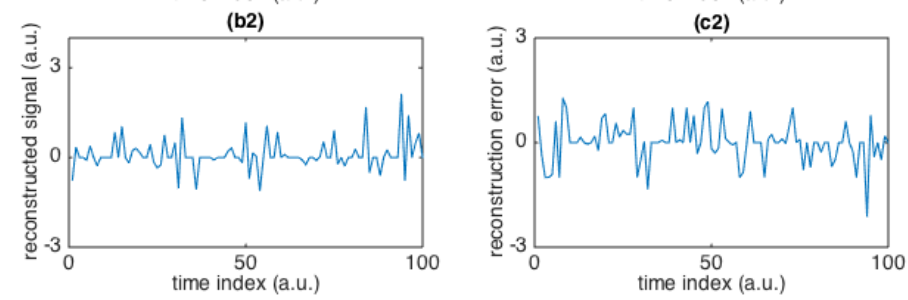

Figure 3 - (a1-a2) Original sparse signal with sparsity level $K=5$ and $K=40$, respectively. (b1-b2) Reconstructed signal using the linear optimization problem described by Eq. (2) and in the context of chaos-based sensing. (c1-c2) Reconstruction error between the two signals for each sparsity level.

However, the deterioration of performance may occur for different sparsity level depending on the type of sensing mechanism used in the linear optimization problem in Eq. (2). Hence, a systematic study comparing the performance between the optimal case of Gaussian sensing and chaotic sensing was performed. For each value of $K \in$ $\llbracket 1,50 \rrbracket$, we have performed a thousand reconstructions for various instantiations of the sparse signal and sensing matrices in the chaotic and Gaussian cases, respectively. The results are shown in Figure 4. We observe that in both cases the transition toward poor reconstruction performance occurs for a similar sparsity level, around $K=15$. The two curves are almost superimposed. This proves that chaotic sensing performs as well as the Gaussian and is therefore quasi-optimal. It also shows that a chaotic-based sensing matrix satisfies empirically the RIP property.

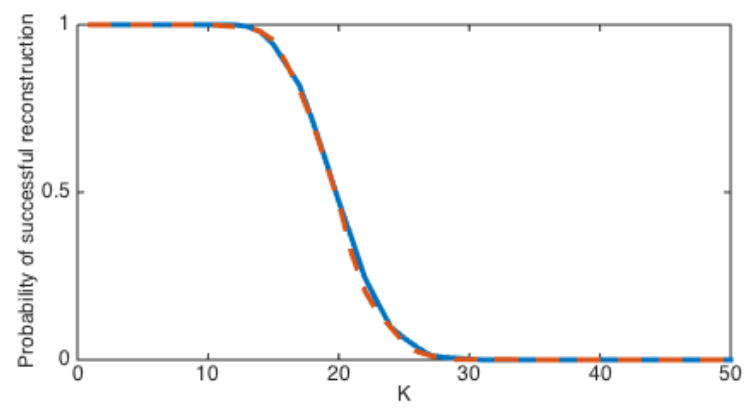

Figure 4 - Reconstruction performance curve showing the probability of successful reconstruction for increasing values of $K$ from 1 to 50. For each value of $K, 1000$ reconstruction are realized with different instantiations of sparse signals and sensing matrices. The Gaussian and chaotic sensing are representing in red dashed lines and solid blue lines respectively. We have used the following numerical values for the reconstruction : $\mathrm{N}=100$ and $\mathrm{M=50}$. 


\section{CONCLUSION}

In this study, we have shown that it was possible to exploit optical chaos for a type of signal processing application that is different from the random bit generation or chaos cryptography. We highlighted that the chaotic intensity values generated by a semiconductor laser with optical feedback can be readily used as elements of a sensing matrix to perform the quasi-perfect reconstruction of sparse signal in the framework of compressive sensing. We have also highlighted that the chaos-based sensing mechanism has a performance comparable to the optimal case of Gaussian random variables. Because of the very large bandwidth of photonics devices, our study provides a first step towards a physical implementation of advanced algorithms for sparse reconstruction at ultra-high speed.

\section{REFERENCES}

[1] Candes, E., Romberg, J. and Tao, T., "Robust uncertainty principles: Exact signal reconstruction from highly incomplete frequency information,” IEEE Trans. Inform. Theory 52, 489 - 509 (2006).

[2] Donoho, D. “Compressed Sensing,” IEEE Trans. Inform. Theory 52, 1289 - 1306 (2006).

[3] Candès, E. and Tao, T., "Near optimal signal recovery from random projections: Universal encoding strategies?" IEEE Trans. Inform. Theory 52(12), 5406 - 5425 (2006).

[4] Lustig, M., Donoho, D.L., and Pauly, J.M., "Sparse MRI: The application of compressed sensing for rapid MR imaging," Magn. Reson. Med. 58(6), 1182 - 1195 (2007).

[5] Gross, D., Liu, Y.-K., Flammia, S. T., Becker, S. and Eisert, J. “Quantum State Tomography via Compressed Sensing," Phys. Rev. Lett. 105, 150401 (2010)

[6] Rivenson, Y., Stern, A. and Rosen, J., "Compressive multiple view projection incoherent holography," Opt. Express $19,6109-6118(2011)$.

[7] Argyris, A., Syvridis, D., Larger, L., Annovazzi-Lodi, V., Colet, P., Fischer, I., Garcia-Ojalvo, J., Mirasso, C. R., Pesquera, L. and Shore, K. A., "Chaos-based communications at high bit rates using commercial fibre-optic links" Nature 437, 343 - 346 (2005).

[8] Uchida, A., Amano, K., Inoue, M., Hirano, K., Naito, S., Someya, H., Oowada, I., Kurashige, T., Shiki, M., Yoshimori, S., Yoshimura, K., and Davis, P., "Fast physical random bit generation with chaotic semiconductor lasers" Nat. Photon. 2, $728-732$ (2008).

[9] Rontani, D., Locquet, A., Sciamanna, M. and Citrin, D. S., "Loss of time-delay signature in the chaotic output of a semiconductor laser with optical feedback," Opt. Lett. 32, 2960 - 2962 (2007).

[10] Rontani, D., Locquet, A., Sciamanna, M., Citrin, D. S., and Ortin, S., "Time-delay identification in a chaotic semiconductor laser with optical feedback: a dynamical point of view," IEEE J. Quantum Electron. 45(7), 879 - 891 (2009).

[11] Li, N., Kim, B., Locquet, A., Choi, D., Pan, W. and Citrin, D. S., "Statistics of the optical intensity of a chaotic external-cavity DFB laser," Opt. Lett. 39, 5949 - 5952 (2014). 
[12] Soriano, M. C., Garcia-Ojalvo, J., Mirasso, C. R., and Fischer, I., "Complex photonics: Dynamics and applications of delay-coupled semiconductors lasers," Rev. Mod. Phys. 85, 421\{470 (2013).

[13] Ohtsubo, J. “Semiconductor Lasers: Stability, Instability and Chaos,” $3^{\text {rd }}$ Ed., Springer-Verlag

[14] Yu, L., Barbot, J.-P., Zheng, G. and Sun, H., “Compressive Sensing With Chaotic Sequence,” IEEE Signal Process. Lett. 7, $731-734$ (2010). 